\title{
Auditory distraction does more than disrupt rehearsal processes in children's serial recall
}

\author{
Angela M. AuBuchon ${ }^{1} \cdot$ Corey I. McGill ${ }^{2}$. Emily M. Elliott ${ }^{2}$
}

Published online: 29 November 2018

(C) Psychonomic Society, Inc. 2018

\begin{abstract}
As children mature, their ability to remember information improves. This improvement has been linked to changes in verbal control processes such as rehearsal. Rehearsal processes are thought to undergo a quantitative shift around 7 years of age; however, direct measurement of rehearsal is difficult. We investigated a measure of rehearsal ability in children and compared this measurement to serial recall performance in the presence of auditory distractors. Theories of auditory distraction effects in children rely upon a combination of attentionally based and serial-order-based processes (Elliott et al. in Journal of Memory and Language, 88, 39-50, 2016); the present work contributes to the understanding of auditory distraction effects by measuring both types of processes within one study. Children completed an individually adjusted serial-recall task with auditory distractors. To assess rehearsal, each child's proportionalized articulatory difference (PAD) score was calculated from performance on adaptive digit span tasks performed in quiet and under articulatory suppression (see also Jarrold \& Citroën in Developmental Psychology, 49, 837-847, 2013). Attentional processes were measured in two ways: first, by using complex span tasks, and second, by children's vulnerability to disruption in the context of irrelevant sound. The results indicated that the rehearsal measure was significantly related to the auditory distraction effect, but this relation was isolated to the attentional-diversion component of the irrelevant-sound effect. The results provide preliminary evidence that children consume attentional resources during rehearsal. Moreover, irrelevant sound disrupts children's rehearsal not solely through automatic, obligatory conflict. Rather, irrelevant sound diverts children's attention, which prevents attentional resources from supporting rehearsal processes.
\end{abstract}

Keywords Rehearsal $\cdot$ Auditory distractions $\cdot$ Serial-order recall $\cdot$ Attention $\cdot$ Children

Rehearsal, the silent repetition of an item's phonological or articulatory code, is one of the many control processes outlined by the Atkinson and Shiffrin (1968) modal memory model. In the 50 years since the model was formulated, the importance of rehearsal for maintaining information in the short-term store has been well documented. Still, the development of rehearsal as a control process is not understood. Previous investigations in rehearsal development have focused on the age at which children begin rehearsing, but the results from these studies have been inconsistent (Flavell, Beach, \& Chinsky, 1966; Gathercole \& Adams, 1994). The continuing controversy about rehearsal's age of onset has established the need for a more nuanced examination of other

Angela M. AuBuchon

angela.aubuchon@boystown.org

1 Boys Town National Research Hospital, Omaha, NE, USA

2 Louisiana State University, Baton Rouge, LA, USA factors that might interact with children's ability to use rehearsal. Of particular interest is whether children rely on attentional control when engaging rehearsal processes (Elliott et al., 2016; Jarrold \& Tam, 2011), unlike adults, whose rehearsal processes occur with minimal attentional resources (Morey, Morey, van der Reijden, \& Holweg, 2013; NavehBenjamin \& Jonides, 1984). The irrelevant-sound paradigm is a powerful tool for examining the interactions of attention and rehearsal. In the irrelevant-sound effect (ISE), the presence of background noise impairs performance during serial-ordered short-term memory tasks (see, e.g., Colle \& Welsh, 1976). Both a failure to control attention and disruption of rehearsal processes have been invoked as explanatory mechanisms for the ISE (Banbury, Macken, Tremblay \& Jones; 2001; Hughes, 2014).

Two accounts have been used to explain the ISE in adults: (1) the unitary account of attention, and (2) the duplexmechanism account. According to the unitary account of attention, irrelevant auditory input disrupts memory performance by capturing attention and redirecting it from the to- 
be-remembered visual information to the incoming auditory signal (Cowan, 1995). The duplex-mechanism account proposes that a second mechanism, interference by process, specifically interferes with serially ordered processes within working memory (Hughes, 2014; Hughes, Vachon, \& Jones, 2007). The underlying premise of the interference-by-process account is that the serial order of sounds is a fundamental characteristic of auditory input and that processing this order information is obligatory. In contrast, order information about visual material must be coded intentionally using control processes within working memory. Because the control process of rehearsal is most effective when items are cycled in a consistent serial order (Atkinson \& Shiffrin, 1968), the interference-by-process account asserts that rehearsal processes are disrupted by the obligatory order-processing of irrelevant auditory input (Hughes, 2014).

The interference-by-process mechanism predicts that if children are not rehearsing, they should have no ISE. Nonetheless, the consistent pattern from prior studies is that children either show a comparable or larger magnitude of the ISE, relative to adults (e.g., Klatte, Lachmann, Schlittmeier, \& Hellbrück, 2010). In no cases have children been shown to have a smaller ISE than adults. The presence of a robust ISE in children suggests either that rehearsal plays less of a role in auditory distraction than is proposed by the duplexmechanism account, or that the relative contributions of attention and rehearsal to serial recall shift across development (e.g., Elliott et al., 2016; Joseph, Hughes, Sörqvist, \&Marsh, 2018). Yet, to date, no attempt has been made to directly measure rehearsal as a predictor for the effects of auditory distraction in children.

\section{Separating the ISE into rehearsal and attentional components}

A common ISE paradigm involves manipulating the amount of variation present within the string of irrelevant sounds. Auditory distractors that change in noticeable ways from one item to the next (changing state) are more disruptive to serial-ordered recall than are tokens that do not change (steady state; Beaman \& Jones, 1997). In adults, the detrimental effect of changing-state distractors has been demonstrated with a variety of stimuli: native-language speech, foreign-language speech, reversed speech, and tones (Jones \& Macken, 1993; Jones, Miles, \& Page, 1990). Because the changing-state component is observed with nonlexical distractors, auditory disruption is attributed to sounds' acoustic - rather than phonological or semantic - properties (but see also Röer, Körner, Buchner, \& Bell, 2017a, b). Specifically, the changing-state component is attributed to increased variation introducing more irrelevant—but automatically processed —order information, which disrupts adults' rehearsal processes (Jones,
Madden, \& Miles, 1992). Converging evidence that irrelevant sounds disrupt adults' rehearsal processes has come from manipulations of articulatory suppression. Articulatory suppression, or the overt repetition of an irrelevant word or syllable ("ba, ba, ba, ba"), is one technique used to prevent rehearsal processes (Baddeley, Thomson, \& Buchanan, 1975; Murray \& Griffiths, 1968). When required to engage in articulatory suppression during an ISE task, adults no longer display a changing-state effect, presumably because the order information in the irrelevant auditory input has nothing to disrupt (Jones, Macken, \& Nicholls, 2004).

Despite showing little to no steady-state effect, adults' serial-recall performance declines in the presence of even a single unexpected deviation from a steady state (e.g., Hughes et al., 2007; Jones, Madden, \& Miles, 1992; Körner, Röer, Buchner, \& Bell, 2017). This deviation effect reflects the attentional-capture component of adult's ISE. It is debated whether the attentional component of the ISE is similar for children and adults (Röer, Bell, Körner, \& Buchner, 2018), or whether children show a larger effect of deviation (Joseph et al., 2018). Although steady-state distractors typically do not impact adults' performance, both changing- and steadystate distractors can disrupt children's serial-recall performance (Elliott, 2002; Elliott et al., 2016). The finding that children are distracted by steady-state sounds is consistent with neuroimaging data describing age-related differences during habituation tasks. Magnetoencephalography (MEG) has revealed that brain activity to a repeated tone (evidence for rapid habituation) decreases quickly in adults, but that the auditory evoked response returns in the presence of a single deviant. In contrast, no decrease in brain activity was observed in 9- to 11-year-old children in response to the same train of repeated tones. In fact, the reduced neural activity associated with habituation was observed only after children had been exposed to 30 trains of the repeated tone (Muenssinger et al., 2013). Also in contrast to adult performance, children experience auditory disruption on the missing-item task, which is devoid of serial-order processing (Elliott et al., 2016; Joseph et al., 2018). Taken together, the evidence provided by Elliott and colleagues (Elliott, 2002; Elliott et al., 2016) - in conjunction with neuroimaging data of children's failure to habituate - indicate that children are especially prone to attentional diversion during the ISE and that, in children, attentional diversion can be indexed by the steady-state component.

\section{The development of rehearsal in young children}

Conclusions regarding rehearsal and children's ISE are complicated by inconsistent descriptions of the developmental trajectory of rehearsal. Articulation rate is the standard estimate of rehearsal speed (Baddeley et al., 1975). Faster rehearsal 
allows for the reactivation of more items before those items fade from memory. Accordingly, relations between articulation rate and memory span are regularly observed in adults (Baddeley et al., 1975; Naveh-Benjamin \& Ayres, 1986). For children 7 to 12 years of age, articulation rate accounts for up to $24 \%$ of the variance in memory span (Cowan et al., 1998). However, for children younger than age 7, a relationship between articulation rate and memory span is rarely observed. Because consistently measurable levels of rehearsal do not appear until age 7, rehearsal abilities are thought to undergo a qualitative shift around this age (Gathercole \& Adams, 1994; Gathercole \& Hitch, 1993).

The exact nature of this shift is under debate. It may represent a marked age of onset, prior to which rehearsal is absent (Gathercole, Adams, \& Hitch, 1994), or a shift in the attentional resources required to engage rehearsal (Guttentag, 1984; Jarrold \& Tam, 2011), or simply a statistical consequence of using methods and scoring procedures that are insensitive to the smaller effect sizes that accompany slower rehearsal (Jarrold \& Tam, 2011). Children younger than 7 years of age do display a recall advantage for lists of short words relative to long words - another marker of rehearsal, known as the word length effect-under specific task conditions (Gathercole \& Adams, 1994; Hitch, Halliday, Dodd, \& Littler, 1989). Additionally, age-related increases in articulation speed from 4 to 10 years of age correspond to group-level increases in memory span, supporting a proposal that rehearsal becomes faster during development and that this increase in rehearsal speed drives memory development (Hitch et al., 1989; Hulme \& Tordoff, 1989). Rehearsal-related changes in serial recall from ages 8 to 12 are accompanied by a freeing of attentional resources for use on a secondary task (Guttentag, 1984). Even by the ages of 11-12, children's serial recall is not fully adult-like, suggesting that rehearsal processes are still developing during the middle school years (Elliott, 2002).

Inconsistencies regarding rehearsal development may be due, in part, to focusing on estimates of children's rehearsal speed rather than their rehearsal efficiency. Adults appear to consume a minimal amount of attentional resources when initiating rehearsal (Morey et al., 2013; Turley-Ames \& Whitfield, 2003), but once a process is initiated, adults' use of verbal processing strategies - including rehearsal-continues automatically (Kowialiewski \& Majerus, 2018; Naveh-Benjamin \& Jonides, 1984). Thus, articulation rate reasonably reflects the limiting factor of speed in adults' rehearsal skills. However, if children rely on attentional resources to initiate as well as to continue rehearsal, then speed is unlikely to be the only contributor to their individual differences.

The need for a more sophisticated measure of children's rehearsal is highlighted by findings from the phonological similarity effect paradigm that have demonstrated that conclusions about children's verbal processing skills are dependent on the scoring procedures used (Jarrold \& Citroën, 2013). The phonological similarity effect refers to the phenomenon that more order errors are produced when recalling lists of phonologically similar words (e.g., bat, bag, mat, man) rather than phonologically distinct words (e.g., bus, clock, girl, hand). The presence of the phonological similarity effect indicates that memory traces are encoded in a phonological form suitable for rehearsal (Hulme \& Tordoff, 1989). When phonological similarity effects are calculated as raw difference scores, they are observed only in groups of children age 7 and older (Hitch, Halliday, Schaafstal, \& Heffernan, 1991). The concern with comparing absolute effect sizes is that baseline performance significantly differs across groups. Jarrold and Citroën argued that the size of the phonological similarity effect scales to baseline performance of short-term memory; therefore, young children should be expected to have smaller phonological similarity effects than either older children or adults. Accordingly, Jarrold and Citroën observed larger absolute effect sizes for older groups, but when they controlled for baseline performance by using a proportionalized scoring method, the effect sizes were equivalent across ages. These findings demonstrate that even children younger than 7 years of age can hold items in a verbal/phonological form, but that verbal processing strategies are not equally beneficial across ages. Moreover, age differences likely reflect underlying limits in storage capacity or attentional resources.

Rehearsal abilities have not been directly assessed in either adults or children in the auditory-distraction literature. When individual differences in the size of the ISE are investigated, they are typically considered with regard to working memory span. This line of research has followed observations that low working memory span individuals are more susceptible to auditory distraction when the unattended channel of a dichotic listening task contains lexically salient information (Conway, Cowan, \& Bunting, 2001). Indeed, complex working memory span measures are often used to index attentional-control processes in adults (Engle, 2002). Nevertheless, working memory span is not a reliable predictor of the ISE in either children (Elliott \& Briganti, 2012) or adults (Beaman, 2004; Körner et al., 2017). However, theories of the relationship between children's working memory capacity and auditory distraction have focused primarily on the changing-state component, which is proposed to occur in response to rehearsal disruption (Elliott \& Briganti, 2012). As we described previously, attentional diversion can be indexed by the steady-state component in children. The potential role of working memory capacity has yet to be elucidated separately for this component.

\section{Purpose of the present study}

The goal of the present study was to better understand how individual differences in attentional control and rehearsal 
contribute to the size of the ISE in a large group of children who, because they fell within a narrow age range near the 7year-old shift, should be rehearsing but were unlikely to be rehearsing at adult levels (Lehmann \& Hasselhorn, 2007, 2012). Of specific interest was (a) whether prior investigations discounting the role of working memory capacity would be replicated even when the ISE was considered in its constituent parts (i.e., the steady-state and changing-state components), and (b) whether rehearsal ability is a reliable predictor of children's ISE, particularly the changing-state component, as is predicted by the interference-by-process account.

To understand whether rehearsal ability predicts the ISE, we first attempted to quantify individual differences in the effectiveness of children's rehearsal by calculating a proportionalized articulatory difference (PAD) score. The PAD score implemented the same scoring method that Jarrold and Citroën (2013) had used to calculate children's verbalization skills, but instead of assessing the effects of phonological similarity, in the present study we estimated the effects of articulatory suppression. Then we assessed whether the PAD score and/or rehearsal speed (as estimated by articulation rate) were related to the size of children's ISE. If the duplex-mechanism account is applied to children in the same manner as adults, then rehearsal speed should be related to the ISE, and this relationship would be driven primarily by the ISE's changing-state component. This prediction is based on children employing adult-like rehearsal processes that, while intentional, are also automatic and limited primarily by rehearsal speed. If, however, children rely on attentional resources while engaging rehearsal, then rehearsal speed is unlikely to be limited primarily by articulation rate. In this case, we would expect the PAD score to predict the ISE better than would rehearsal speed. Moreover, we would expect rehearsal and attention to interact via the steady-state component of the ISE. The latter prediction would be consistent with a recent interpretation of the duplex-mechanism account in which rehearsal and attentional processes interact in children in ways that are different from those that occur in adults (Elliott et al., 2016; Joseph et al., 2018).

\section{Method}

\section{Participants}

Thirty-four children, ages 7 years 2 months to 8 years 10 months $(7 ; 2$ to $8 ; 10)$ were recruited from the community surrounding a large state university; an additional 33 children, ages $7 ; 0$ to $8 ; 7$, were recruited from an existing participant database at a hospital-based research center. Two children from the first site and three children from the second did not wish to complete all of the tasks. Each site also experienced one computer/experimenter error. One child $(9 ; 5)$ was not included in the dataset due to being outside the recruited age range. Thus, the final dataset included 60 children, ages 7;0 to 8;7. All children had normal or corrected-to-normal vision, suffered no hearing loss, and were native monolingual English speakers, with the exception of one child who was a bilingual English-French speaker.

\section{Procedure}

All procedures were approved by the local institutional review boards. All testing occurred during a single visit that lasted between 50 and $80 \mathrm{~min}$. The auditory stimuli were presented over headphones at comfortable listening levels $(\sim 60 \mathrm{~dB}$ SPL). Children made all responses aloud, and their verbal responses were entered immediately by the experimenter. The order of testing was counting span, listening span, articulation rate, digit span in quiet, digit span under articulatory suppression, and ISE task.

\section{Materials}

Measures of working memory These included a counting span task and a listening span task. Counting span was adapted from Cowan et al. (2003). Children were presented with visual arrays comprising two to nine target shapes (blue squares) and two to nine distractors (red triangles). Children silently counted the number of targets while ignoring the distractors, then reported the number of targets aloud. After two to five arrays, the child was asked to recall, in order, the responses they had provided to the immediately preceding arrays.

In the listening span task (Daneman \& Carpenter, 1980; Kail \& Hall, 1999), children heard 60 declarative sentences, half of which were clearly true (e.g., "Milk comes from cows.") or not true (e.g., "Toads live in a couch."). Following each sentence, the child stated whether or not the sentence made sense and then repeated the last word of the sentence. After one to five sentences, the child was asked to recall, in order, the final word of each of the sentences.

Each working memory measure was scored as the number of items recalled in the correct serial position. The two scores were correlated, $r(58)=.47, p<.01$; therefore, they were converted into $z$-scores, which were averaged to create a working memory composite score.

Articulation rate To minimize any potential influence of motor programming and speech output, articulation rate was measured with a highly familiar string of items. Children were instructed to count from 1 to 10 as quickly as possible. They repeated this task three times while being recorded. The beginning and end of each utterance were marked using the Audacity software, and the total length of each utterance was calculated. Articulation rate was scored as the average utterance length across the three repetitions. 
Digit span tasks Participants completed two adaptive digit span tasks: one in quiet and one under articulatory suppression. Participants were shown a series of digits from the set of 1-9 in the center of the computer monitor. The digits were presented at the rate of one per second, and no digit was repeated within a given list. After the final item, participants were instructed to reproduce the list of items aloud. Four lists were presented at each length. If a child accurately recalledusing a strict serial-order criterion-at least two of the four lists, then the list length was increased by one item. If the child accurately recalled fewer than two of the four lists, the task was terminated.

For digit span in quiet, the initial list length was three digits. Each child received two scores based on their performance: (1) Integer span reflected the longest list length for which the child was able to accurately reproduce at least two lists. (2) Cumulative score was the longest list length for which the child correctly recalled all four lists (with a minimum longest list length set to 2 ), plus 0.25 for every list correctly recalled from a longer list length (i.e., digit span $=[$ highest list length for which all four lists were correct $]+0.25 *$ [\# of subsequent lists correctly recalled]).

During digit span under articulatory suppression, the children were asked to recite the nonsense syllable "da" (adapted from Fatzer \& Roebers, 2012), at the rate of two per second, from the moment the trial was initiated until the recall instructions appeared. To mitigate potential floor effects, digit span under articulatory suppression had an initial list length of two digits. However, due to computer error, three children completed the digit span in quiet task a second time with articulatory suppression instructions administered verbally. Thus, for these three children, the initial list length was three. A cumulative score was calculated for digit span under articulatory suppression in the same manner as for digit span in quiet with the exception that the minimum longest list length was set to 1 .

A proportionalized articulatory difference (PAD) score [(digit span in quiet - digit span under articultory supporession)/digit span in quiet] was calculated using Jarrold and Citroën's (2013) formula for examining children's phonological similarity effect. By using the cumulative scores from digit span both in quiet and under articulatory suppressionwith the cumulative score for digit span in quiet as the baseline - the PAD score can be interpreted as estimating the extent to which articulatory suppression disrupts rehearsal. A PAD score near 0 indicates that a child performed comparably in quiet and under suppression, suggesting that rehearsal was not utilized. PAD scores near 1 indicate greater disruption due to articulatory suppression, and thus more reliance on rehearsal.

Irrelevant-sound effect task This task required children to remember a sequence of visually presented digits while sometimes also ignoring auditory distractors. The to-beremembered digits were presented in the center of the computer screen at a rate of one per second. No digit was repeated during a given list. After the final digit, participants were instructed to reproduce the list of digits aloud. To ensure that the allocation of attentional resources was not influenced by capacity constraints (Cowan, Morey, AuBuchon, Zwilling, \& Gilchrist, 2010), individual differences were controlled by using each child's integer span score from digit span in quiet to determine the list length on the ISE task.

The ISE task had ten blocks of three trials (30 trials total). In each block, one trial came from the steady-state auditory condition, during which participants heard the word red repeated for the duration of digit presentation; one trial came from the changing-state auditory condition, during which participants heard a random selection of the words big, long, short, tall, blue, green, white, red, and yellow during digit presentation. The remaining trial occurred in silence. During each block, auditory condition (steady-state, changing-state, silence) was randomly ordered. In both conditions with distractors, the onsets of the auditory stimuli were simultaneous with the onsets of the to-be-recalled digits. Performance was based on the number of digits recalled in the correct position within each auditory condition. From these scores, we could then calculate (1) the overall ISE [silence - changing-state] (2) the steady-state component [silence - steady-state], and (3) the changing-state component [steady-state - changing-state].

\section{Statistical analyses}

Statistical analyses were performed using R Version 3.4.2 ( $\mathrm{R}$ Core Team, 2017) and the packages tidyr (Wickham \& Henry, 2018), DescTools (Signorell, 2018), sjstats (Lüdecke, 2018), and $\mathrm{lm}$.beta (Behrendt, 2014). A within-subjects analysis of variance (ANOVA) was used to confirm that the present study replicated previously described ISE patterns observed in children. Specifically, we expected recall during silence to be greater than recall during steady-state and changing-state distractors, which should also differ. To assess the relation between rehearsal and the ISE, we created a linear regression model including age, working memory, PAD, and articulation rate as predictors. Our secondary goal was to determine whether any interference to children's rehearsal due to auditory distraction resembled adult-like patterns. Specifically, the interference-by-process account stipulates that disruption to adults' rehearsal occurs in the presence of changing-state distractors. To test this claim, we parsed the ISE into its component parts - the steady-state component (silence - steady state) and the changing-state component (steady state - changing state) - and tested each component with the same model of predictors. If children's ISE were adult-like, then rehearsal abilities should predict the changingstate but not the steady-state component. Data collection site did not contribute to the three regression models and was, therefore, omitted from the final models. 


\section{Results}

Descriptive statistics are presented in Table 1, and a correlation matrix of all variables is presented in Table 2. Descriptive statistics for the ISE tasks are reported as proportions correct in order to better retain meaning across the children's differing list lengths. However, since each child acted as his or her own control, all analyses were done with absolute values. The scores for the ISE task during silence, steady-state sounds, and changing-state sounds were analyzed using a withinsubjects ANOVA. The results of the ANOVA indicated a significant main effect of auditory condition, $F(2,118)=82.8, p$ $<.01, \eta_{\mathrm{p}}{ }^{2}=.58, M S E=27.7$, on the number of digits recalled in the correct serial position. All three conditions differed significantly after making Benjamini-Hochberg corrections, which decrease the false discovery rate while retaining the power to detect small effects, on the pairwise comparisons. Performance in silence ( $M=32.5, S D=6.8)$ was significantly higher than performance during steady-state $(M=28.2, S D=$ $7.7, p<.01)$ and changing-state $(M=20.3, S D=8.1, p<.01)$ distraction, and the latter scores also differed from one another $(p<.01)$. Each participant's absolute scores were transformed into difference scores representing the size of the ISE (silence - changing state), size of the steady-state component (silence - steady state), and size of the changing-state component (steady state - changing state) for the regression analysis.

The first regression model assessed the contributions of age, working memory, PAD score, and articulation rate to the size of children's ISE. The model was significant $[F(4$, $55)=4.37, p<.01]$, and together, these four variables accounted for $24 \%$ of the variance observed in the ISE (Table 3). Notably, only the PAD score was a significant predictor $(\beta=.50, p<.01)$. One potential limitation of the model is that the ISE task utilized the same stimuli (digits) and general methodology (serial recall) as the constituent tasks (digit span in silence and digit span under articulatory suppression) that formed the PAD score. If the task demandsrather than PAD's ability to estimate rehearsal - were driving the observed relationship, we would expect scores on both span tasks to substitute equally well for the PAD score. When the PAD score was replaced with cumulative scores for digit span in quiet (which permitted the use of rehearsal), the regression equation remained significant $[F(4,55)=6.73$, $p<.01]$, with an $R^{2}$ of .33, and digit span in quiet was the only significant predictor $(\beta=.64, p<.01)$. However, when the PAD score was replaced with the cumulative score for digit span under articulatory suppression (during which rehearsal was presumably blocked or limited), the regression equation was no longer significant $\left[F(4,55)=0.31, p=.87, R^{2}=.02\right]$. These findings limit concerns that the correlation between PAD and the ISE is simply due to commonalities between digit span and the ISE task, such as shared stimuli or the shared dual-task nature of recalling under articulatory suppression and recalling in the presence of background noise.

Separate regression models were then created for each component of the ISE: the steady-state component (silence steady state) and changing-state component (steady state changing state). The purpose of these analyses was to determine whether children's ISE conformed to expectations set forth in the adult ISE literature, specifically as detailed by the duplex-mechanism account. If so, then any relationship with rehearsal would be confined to the changing-state effect, per the interference-by-process explanation. Instead, the regression model significantly predicted the steady-state effect

Table 1 Descriptive statistics $(N=60)$

\begin{tabular}{|c|c|c|c|}
\hline Task & Mean $(S D)$ & Min & Max \\
\hline Age (years) & $7.8(0.4)$ & 7.0 & 8.6 \\
\hline Working memory composite & $0(0.9)$ & -2.3 & 1.8 \\
\hline Counting span & $34.3(10.0)$ & 6 & 57 \\
\hline Listening span & $14.8(8.2)$ & 1 & 35 \\
\hline Articulation rate (seconds) & $1.9(0.3)$ & 1.4 & 3.0 \\
\hline Proportionalized articulatory difference score & $.29(.18)$ & -.15 & .65 \\
\hline Digit span in silence & $4.14(0.92)$ & 2.0 & 6.5 \\
\hline Digit span under articulatory suppression & $2.82(0.58)$ & 1.75 & 4.0 \\
\hline \multicolumn{4}{|l|}{ Irrelevant-sound effect task (proportions correct) } \\
\hline Silence & $.76(.17)$ & .38 & 1.0 \\
\hline Steady-state sound & $.67(.23)$ & .20 & 1.0 \\
\hline Changing-state sound & $.49(.24)$ & .06 & 1.0 \\
\hline
\end{tabular}

Standard deviations $(S D \mathrm{~s})$ are in parentheses. Working memory composite reflects the average $z$-scores of counting span and listening span. Counting span and listening span are scored as the total number of items recalled in the correct serial position. Articulation rate is the average time (in seconds) to recite the digits 1 to 10 . Scores for the irrelevant-sound effect are transformed into proportions correct here, but the analyses were conducted with absolute values 
Table 2 Pairwise correlations for the irrelevant-sound effect (and its component effects) and predictor variables

\begin{tabular}{llllllllll}
{$[1]$} & {$[2]$} & {$[3]$} & {$[4]$} & {$[5]$} & {$[6]$} & {$[7]$} & {$[8]$} & {$[9]$} & {$[10]$} \\
\hline
\end{tabular}

[1] Age (years)

[2] Working memory composite

[3] Counting span

[4] Listening span

[5] Articulation rate

[6] PAD score

[7] Digit span (silence)

[8] Digit span (articulatory suppression)

[9] Irrelevant-sound effect

[10] Steady-state effect

[11] Changing-state effect

\begin{tabular}{rrrrrrrrrr}
.15 & & & & & & & & \\
.11 & $.86^{*}$ & & & & & & & \\
.15 & $.86^{*}$ & $.47^{*}$ & & & & & & \\
-.20 & -.20 & -.22 & -.14 & & & & & \\
.19 & .12 & .05 & .16 & -.18 & & & & \\
$.34^{*}$ & $.31^{*}$ & .24 & $.29^{*}$ & $-.31^{*}$ & $.65^{*}$ & & & \\
.08 & .22 & $.27^{*}$ & .10 & -.12 & $-.53^{*}$ & .24 & & \\
.08 & .00 & -.05 & .04 & -.05 & $.49^{*}$ & $.53^{*}$ & -.10 & & \\
.06 & -.02 & -.04 & .00 & -.22 & $.41^{*}$ & $.42^{*}$ & -.13 & $.59^{*}$ & \\
.03 & .01 & -.02 & .04 & .16 & .15 & .19 & .01 & $.56^{*}$ & $-.33^{*}$ \\
\hline
\end{tabular}

${ }^{*} p<.05$, uncorrected for multiple comparisons

$[F(4,55)=3.46, p=.01]$, with an $R^{2}$ of .20 , and the variables included in this model were unable to account for any additional decrements in performance due to the changing-state nature of the stimuli (Table 4).

\section{Discussion}

The present study extended previous findings that the underlying mechanisms of the ISE are different in children than in adults, and in doing so, it has provided important insights into the developmental trajectory of rehearsal processes. First, we replicated previous findings that children ages 7 and 8 display both steady-state and changing-state components within an ISE paradigm (Elliott, 2002, Elliott et al., 2016; Joseph et al., 2018; Röer et al., 2018). We then explored which individual differences of age, working memory span, articulation rate (as an estimate of rehearsal speed), or PAD score (as an estimate of how effectively children used rehearsal to increase digit span) best predicted the size of children's ISE. This was the first study, to our knowledge, to attempt to measure individual differences in children's rehearsal abilities and to relate them to the different components of auditory distraction.

Table 3 Summary of simple regression analyses for variables predicting the irrelevant-sound effect $(N=60)$

\begin{tabular}{lrcc}
\hline Variable & \multicolumn{1}{l}{ B } & SE $B$ & $\beta$ \\
\hline Age & -0.02 & 2.27 & -.001 \\
Working memory & -0.54 & 1.15 & -.06 \\
PAD & 21.98 & 5.36 & $.50^{*}$ \\
Articulation rate & 0.66 & 3.29 & .02 \\
$R^{2}$ & & .24 & \\
$F$ & & $4.37^{*}$ & \\
\hline${ }^{*} p<.01$ & &
\end{tabular}

According to the duplex-mechanism account, the changing-state component of the ISE occurs because auditory distraction interferes with adults' ability to utilize rehearsal strategies while maintaining the serial order of items in short-term memory (Hughes, 2014). Consistent with this claim, children's rehearsal abilities predicted the size of the overall ISE. However, if the rehearsal abilities of these 7- and 8-year-olds (who had passed the 7-year age mark for when the qualitative shift in rehearsal is regularly observed) had been adult-like, we would have expected their rehearsal speed to be related to the overall ISE. Instead, the PAD score (as conceptualized by the degree to which articulatory suppression disrupted performance on a separate digit span task) was the sole significant predictor in the model.

The relation between PAD and the size of the ISE indicates that auditory distraction disrupts rehearsal in children, which provides converging evidence that the duplex-mechanism account can be extended to children (see Elliott et al., 2016; Joseph et al., 2018). However, such an extension comes with a caveat, discussed in more detail below. Additionally, the usefulness of PAD, but not articulation rate, as a predictor raises concerns regarding the use of articulation rate as an

Table 4 Summary of simple regression analyses for variables predicting the irrelevant-sound effect $(N=60)$

\begin{tabular}{|c|c|c|c|c|c|c|}
\hline \multirow[t]{2}{*}{ Variable } & \multicolumn{3}{|c|}{ Steady-state effect } & \multicolumn{3}{|c|}{ Changing-state effect } \\
\hline & $B$ & $S E B$ & $\beta$ & $B$ & $S E B$ & $\beta$ \\
\hline Age & -0.60 & 2.04 & -.04 & 0.57 & 2.16 & .04 \\
\hline Working memory & -0.80 & 1.03 & -.10 & 0.25 & 1.09 & .03 \\
\hline PAD & 15.27 & 4.81 & $.40^{*}$ & 6.71 & 5.09 & .18 \\
\hline Articulation rate & -4.16 & 2.96 & -.18 & 4.82 & 3.13 & .21 \\
\hline$R^{2}$ & & .20 & & & .06 & \\
\hline$F$ & & $3.46^{*}$ & & & 0.92 & \\
\hline
\end{tabular}


estimate of children's rehearsal abilities. Because both articulation rate and PAD are considered estimates of rehearsal, we might have expected either (1) both to be significant predictors in the model, or (2) the two to be highly correlated and their shared variance to obscure each other's contributions. In fact, even once PAD was removed, articulation rate $(\beta=-.04, p=$ $.75)$ was not a significant predictor of the $\operatorname{ISE}[F(3,56)=0.16$, $\left.p=.92, R^{2}=.01\right]$. Articulation rate is often used to estimate rehearsal speed because rehearsal is assumed to depend on the articulatory pathway (Awh et al., 1996) as well as on the spoken length of the stimuli (Baddeley et al., 1975). However, this logic only follows if participants are actually rehearsing, and doing so with efficiency similar to that when they produce spoken language. Considering the age range of the participants $(7 ; 0$ to $8 ; 7)$ and the observed range of PAD scores ( -.15 to .65$)$, it is possible that some of the children in the present study did have nearly adult-like levels of rehearsal. Yet some may not have begun rehearsing at all. Many children are likely situated in-between; they may be rehearsing, but their rehearsal abilities are less developed. For these children, rehearsal may be slower and more attention-demanding than overt speech production. Thus, for many of the children in the present study, articulation rate was likely an insensitive measure of rehearsal ability. We propose that the PAD score, modeled on a proportionalized scoring procedure originally used to examine the phonological similarity effect (Jarrold \& Citroën, 2013), is more sensitive to individual differences in children's rehearsal.

Neither age nor working memory capacity, as measured by two complex span tasks, contributed predictive value to the model of the size of the children's ISE. Children between the ages of 7 and 9 were selected precisely because we anticipated this would be a narrow age range in which children would still display variability in rehearsal development. Incidentally, within this range, age and PAD scores were not significantly correlated $(r=.19, p=.13)$, highlighting that rehearsal development is not strictly age-dependent and that future research should consider the factors that lead to individual variability in rehearsal development.

Prior investigations into individual differences in the ISE have focused on the role of working memory capacity because other paradigms (e.g., dichotic listening) have revealed greater distractibility among low-span individuals. Consistent with those early ISE results (e.g., Beaman, 2004; Elliott \& Briganti, 2012), the present study failed to yield significant findings. Moreover, the design choice of individualizing each child's set size during the ISE task according to his or her digit span integer score likely controlled for some individual variation in working memory capacity; for these reasons, it is unsurprising that working memory capacity was not a useful predictor of children's ISE.

Although rehearsal use (i.e., PAD) significantly contributed to the model, the amount of variance it predicted was only
$24 \%$. Additionally, visual analysis of the model's predicted ISE as compared to children's actual ISE revealed that the model underpredicted ISE at the high end of the range and overpredicted ISE at the low end of the range. A limitation of the present study was not including a separate and explicit measure of attentional control that could be used as a predictor in the model but was independent of working memory capacity. Such a measure might improve the model fit and better elucidate the relative contributions of attention and rehearsal in children's ISE. Moreover, the use of an explicit measure of attentional control would make it feasible to include an adult comparison group for whom the steady-state effect is not a meaningful measure of attentional control.

To determine whether the effects of auditory distraction on children's rehearsal mirrored adult-like mechanisms, as outlined by the duplex-mechanism account, we separately examined the two components of the ISE: the steady-state component and the changing-state component. For adults, the changing nature of the auditory distractors is what automatically disrupts the use of rehearsal during serial-recall tasks via interference by process. Thus, on the basis of the adult literature, we would predict that rehearsal is related to the changingstate rather than the steady-state component. Contrary to this prediction, the relationship between children's ISE and rehearsal was driven by the steady-state rather than the changing-state component. The steady-state component is often associated with attentional diversion in children (e.g., Elliott, 2002; Elliott et al., 2016; although see Joseph et al., 2018). The finding that PAD scores contributed to the steadystate component suggested that the children who most benefit from rehearsal are also the most prone to attentional diversion. In other words, the 7- to 9-year-old children who rehearse most successfully appear to deplete their attention control resources in the process; thus, even simple auditory distractions such as steady-state sounds are sufficient to disrupt their otherwise successful rehearsal. This interpretation aligns with the duplex-mechanism account, in that both attention and rehearsal are disrupted. However, the degree to which the interference-by-process mechanism can be extended to children is unclear. The present data-in combination with previous findings that auditory distraction disrupts children's missing span performance (Elliot et al., 2016) — suggests that direct competition between the two serially ordered processes may not be the primary mechanism explaining this relation.

\section{Summary}

Atkinson and Shiffrin (1968) originally stipulated that using rehearsal required a trade-off of resources that limited the use of other control processes. However, more recent evidence has indicated that adults' use of rote rehearsal processes is highly automatic and can be used simultaneously with other maintenance processes (Morey et al., 
2013; Naveh-Benjamin \& Jonides, 1984). Still, the process by which children come to have adult-like rehearsal is unknown. Prior to age 7, children show only inconsistent evidence that they rehearse. Even after age 7, children's use of rehearsal does not appear adult-like. The present study provides supporting evidence that rehearsal does not emerge in a highly automatic, adult-like fashion. The observation that the amount of disruption that children experience during the steady-state component is related to successful rehearsal on a separate task indicates that children consume attentional resources even when rehearsing; consequently, tapping attentional resources simply to maintain short-term serial order depletes children of resources to control attention in the presence of auditory distractors. Thus, even rote rehearsal limits their use of other control processes (Atkinson \& Shiffrin, 1968; Elliott et al., 2016; Joseph et al., 2018). It also indicates that rehearsal speed is insufficient to capture the benefits that children receive to memory span when rehearsal is available. This raises questions about what underlying cognitive skills contribute to individual differences in children's rehearsal, including whether children have the metacognitive skills to decide when rehearsal is beneficial (Atkinson \& Shiffrin, 1968). Additionally, other underlying skills, such as processing speed or language ability, may feed into rehearsal (AuBuchon, Pisoni, \& Kronenberger, 2015).

The present study also adds to our growing understanding of the sources of auditory disruption in children. For both theoretical and applied reasons, we need to understand whether children respond to these auditory stimuli in the same way adults do. Children are often faced with the task of learning in noisy environments (Nelson \& Soli, 2000). Children in the age range tested here regularly face independent-learning situations in which they are expected to make sense of visual input, such as reading textbooks, despite the presence of noise from classmates, or even outside events (e.g., lawn mowers or construction). Younger children must regularly attempt to identify target auditory input, such as a teacher's voice amid chattering students. This challenge cannot be fully explained by the overlapping auditory sensory signals, and likely includes contributions from central, cognitive mechanisms (Schafer, 2010). Identifying and describing the mechanisms involved in children's ISE will provide insight into how to minimize the negative impacts of background noise, as well as help teachers and clinicians identify children who are most at risk for learning disruptions due to background noise.

Author note Data and analysis code from this study can be found at https:// osf.io/dca8k/?view_only=39c68db13c3b4f63b85a704c64d28faf and https:// osf.io/wb2xn/?view_only=7f5b050e61744dbe948c5499a56d8382, respectively. Participant recruitment was partially supported by the National Institute of General Medical Sciences of the National Institutes of Health under award number P30DC004662. The authors have no conflicts of interest to disclose. The authors thank the participants for their time, and Grace Meissner for assisting with data collection.

Publisher's Note Springer Nature remains neutral with regard to jurisdictional claims in published maps and institutional affiliations.

\section{References}

Atkinson, R. C., \& Shiffrin, R. M. (1968). Human memory: A proposed system and its control processes. In K. W. Spence \& J. T. Spence (Eds.), The psychology of learning and motivation: Advances in research and theory (pp. 89-195). New York, NY: Academic Press.

AuBuchon, A. M., Pisoni, D. B., \& Kronenberger, W. G. (2015). Verbal processing speed and executive functioning in long-term cochlear implant users. Journal of Speech, Language, and Hearing Research, $58,151-162$.

Awh, E., Jonides, J., Smith, E. E., Schumacher, E. H., Koeppe, R. A., \& Katz, S. (1996). Dissociation of storage and rehearsal in verbal working memory: Evidence from positron emission tomography. Psychological Science, 7, 25-31.

Baddeley, A. D., Thomson, N., \& Buchanan, M. (1975). Word length and the structure of short-term memory. Journal of Verbal Learning and Verbal Behavior, 14, 575-589. https://doi.org/10.1016/S00225371(75)80045-4

Banbury, S. P., Macken, W. J., Tremblay, S., \& Jones, D. M. (2001). Auditory distraction and short-term memory: Phenomena and practical implications. Human Factors, 43, 12-29

Beaman, C. P. (2004). The irrelevant sound phenomenon revisited: What role for working memory capacity? Journal of Experimental Psychology: Learning, Memory, and Cognition, 30, 1106-1118. https://doi.org/10.1037/0278-7393.30.5.1106

Beaman, C. P., \& Jones, D. M. (1997). Role of serial order in the irrelevant speech effect: Tests of the changing-state hypothesis. Journal of Experimental Psychology: Learning, Memory, and Cognition, 23, 459-471. https://doi.org/10.1037/0278-7393.23.2.459

Behrendt, S. (2014). 1m.beta: Add standardized regression coefficients to lm-objects (R package version 1.5-1). https://CRAN.R-project.org/ package $=\operatorname{lm}$. beta

Colle, H. A., \& Welsh, A. (1976). Acoustic masking in primary memory. Journal of Verbal Learning \& Verbal Behavior, 15, 17-31. https:// doi.org/10.1016/S0022-5371(76)90003-7

Conway, A. R. A., Cowan, N., \& Bunting, M. F. (2001). The cocktail party phenomenon revisited: The importance of working memory capacity. Psychological Bulletin \& Review, 8, 331-335.

Cowan, N. (1995). Attention and memory: An integrated framework (Oxford Psychology Series, Vol. 26). New York, NY: Oxford University Press.

Cowan, N., Morey, C. C., AuBuchon, A. M., Zwilling, C. E., \& Gilchrist, A. L. (2010). Seven-year-olds allocate attention like adults unless working memory is overloaded. Developmental Science, 13, 120-133.

Cowan, N., Towse, J. N., Hamilton, Z., Saults, J. S., Elliott, E. M., Lacey, J. F., ... Hitch, G. J. (2003). Children's working-memory processes: A response-timing analysis. Journal of Experimental Psychology: General, 132, 113-132.

Cowan, N., Wood, N. L., Wood, P. K., Keller, T. A., Nugent, L. D., \& Keller, C. V. (1998). Two separate verbal processing rates contributing to short-term memory span. Journal of Experimental Psychology: General, 127, 141-160. https://doi.org/10.1037/00963445.127.2.141

Daneman, M., \& Carpenter, P. A. (1980). Individual differences in working memory and reading. Journal of Verbal Learning and Verbal Behavior, 19, 450-466. https://doi.org/10.1016/S0022-5371(80) 90312-6 
Elliott, E. M. (2002). The irrelevant-speech effect and children: Theoretical implications of developmental change. Memory \& Cognition, 30, 478-487. https://doi.org/10.3758/BF03194948

Elliott, E. M., \& Briganti, A. M. (2012). Investigating the role of attentional resources in the irrelevant speech effect. Acta Psychologica, 140, 64-74. https://doi.org/10.1016/j.actpsy.2012.02.009

Elliott, E. M., Hughes, R. W., Briganti, A., Joseph, T. N., Marsh, J. E., \& Macken, B. (2016). Distraction in verbal short-term memory: Insights from developmental differences. Journal of Memory and Language, 88, 39-50. https://doi.org/10.1016/j.jml.2015.12.008

Engle, R. W. (2002). Working memory capacity as executive attention. Current Directions in Psychological Science, 11, 19-23. https://doi. org/10.1111/1467-8721.00160

Fatzer, S. T., \& Roebers, C. M. (2012). Language and executive functions: The effect of articulatory suppression on executive functioning in children. Journal of Cognition and Development, 13, 454 472. https://doi.org/10.1080/15248372.2011.608322

Flavell, J. H., Beach, D. R., \& Chinsky, J. M. (1966). Spontaneous verbal rehearsal in a memory task as a function of age. Child Development, 37, 283-299.

Gathercole, S. E., \& Adams, A.-M. (1994). Children's phonological working memory: Contributions of long-term knowledge and rehearsal. Journal of Memory and Language, 33, 672-688.

Gathercole, S. E., Adams, A.-M., \& Hitch, G. J. (1994). Do young children rehearse? An individual-differences analysis. Memory \& Cognition, 22, 201-207. https://doi.org/10.3758/BF03208891

Gathercole, S. E., \& Hitch, G. J. (1993). Developmental changes in shortterm memory: A revised working memory perspective. Theories of Memory, 1, 189-209.

Guttentag, R. E. (1984). The mental effort requirement of cumulative rehearsal: A developmental study. Journal of Experimental Child Psychology, 37, 92-105.

Hitch, G. J., Halliday, M. S., Dodd, A., \& Littler, J. E. (1989). Development of rehearsal in short-term memory: Differences between pictorial and spoken stimuli. British Journal of Developmental Psychology, 7, 347-362. https://doi.org/10.1111/j. 2044-835X.1989.tb00811.x

Hitch, G. J., Halliday, M. S., Schaafstal, A. M., \& Heffernan, T. M. (1991). Speech, "inner speech," and the development of shortterm memory: Effects of picture-labelling on recall. Journal of Experimental Child Psychology, 51, 220-234. https://doi.org/10. 1016/0022-0965(91)90033-O

Hughes, R. W. (2014). Auditory distraction: A duplex-mechanism account. PsyCh Journal, 3, 30-41. https://doi.org/10.1002/pchj.44

Hughes, R. W., Vachon, F., \& Jones, D. M. (2007). Disruption of shortterm memory by changing and deviant sounds: Support for a duplex-mechanism account of auditory distraction. Journal of Experimental Psychology: Learning, Memory, and Cognition, 33, 1050-1061. https://doi.org/10.1037/0278-7393.33.6.1050

Hulme, C., \& Tordoff, V. (1989). Working memory development: The effects of speech rate, word length, and acoustic similarity on serial recall. Journal of Experimental Child Psychology, 47, 72-87. https://doi.org/10.1016/0022-0965(89)90063-5

Jarrold, C., \& Citroën, R. (2013). Reevaluating key evidence for the development of rehearsal: Phonological similarity effects in children are subject to proportional scaling artifacts. Developmental Psychology, 49, 837-847. https://doi.org/10.1037/a0028771

Jarrold, C., \& Tam, H. (2011). Cognitive development and working memory: A dialogue between neo-Piagetian theories and cognitive approaches (P. Barrouillet \& V. Gaillard, Eds.). New York, NY: Psychology Press.

Jones, D. M., \& Macken, W. J. (1993). Irrelevant tones produce an irrelevant speech effect: Implications for phonological coding in working memory. Journal of Experimental Psychology: Learning, Memory, and Cognition, 19, 369-381. https://doi.org/10.1037/ 0278-7393.19.2.369
Jones, D. M., Macken, W. J., \& Nicholls, A. P. (2004). The phonological store of working memory: Is it phonological and is it a store? Journal of Experimental Psychology: Learning Memory and Cognition, 30, 656674. https://doi.org/10.1037/0278-7393.30.3.656

Jones, D. M., Miles, C., \& Page, J. (1990). Disruption of proofreading by irrelevant speed: Effects of attention, arousal or memory? Applied Cognitive Psychology, 4, 89-108.

Jones, D., Madden, C., \& Miles, C. (1992). Privileged access by irrelevant speech to short-term memory: The role of changing state. Quarterly Journal of Experimental Psychology, 44A, 645-669. https://doi.org/10.1080/14640749208401304

Joseph, T. N., Hughes, R. W., Sörqvist, P., \& Marsh, J. E. (2018). Differences in auditory distraction between adults and children: A duplex-mechanism approach. Journal of Cognition, 1, 15:1-11. https://doi.org/10.5334/joc. 15

Kail, R., \& Hall, L. K. (1999). Sources of developmental change in children's word-problem performance. Journal of Educational Psychology, 91, 660-668. https://doi.org/10.1037/0022-0663.91.4.660

Klatte, M., Lachmann, T., Schlittmeier, S., \& Hellbrück J., (2010). The irrelevant sound effect in short-term memory: Is there developmental change? European Journal of Cognitive Psychology, 22, 11681191. https://doi.org/10.1080/09541440903378250

Körner, U., Röer, J. P., Buchner, A., \& Bell, R. (2017). Working memory capacity is equally unrelated to auditory distraction by changingstate and deviant sounds. Journal of Memory and Language, 96, 122-137. https://doi.org/10.1016/j.jml.2017.05.005

Kowialiewski, B., \& Majerus, S. (2018). The non-strategic nature of linguistic long-term memory effects in verbal short-term memory. Journal of Memory and Language, 101, 64-83.

Lehmann, M., \& Hasselhorn, M. (2007). Variable memory strategy use in children's adaptive intratask learning behavior: Developmental changes and working memory influences in free recall. Child Development, 78, 1068-1082.

Lehmann, M., \& Hasselhorn, M. (2012). Rehearsal dynamics in elementary school children. Journal of Experimental Child Psychology, 111, 552-560. https://doi.org/10.1016/j.jecp.2011.10.013

Lüdecke, D. (2018). sjstats: Statistical functions for regression models (R package version 0.15.0). Retrieved from https://CRAN.R-project. org $/$ package $=$ sjstats

Morey, C. C., Morey, R. D., van der Reijden, M., \& Holweg, M. (2013). Asymmetric cross-domain interference between two working memory tasks: Implications for models of working memory. Journal of Memory and Language, 69, 324-348. https://doi.org/10.1016/j.jml. 2013.04.004

Muenssinger, J., Stingl, K.T., Matuz, T., Binder, G., Ehehalt, S., \& Preissl, H. (2013). Auditory habituation to simple tones: Reduced evidence for habituation in children compared to adults. Frontiers in Human Neuroscience, 7, 377:1-7.

Murray, D. J., \& Griffiths, E. (1968). Articulation and acoustic confusability in short-term memory. Journal of Experimental Psychology, 78, 679-684.

Naveh-Benjamin, M., \& Ayres, T. J. (1986). Digit span, reading rate, and linguistic relativity. The Quarterly Journal of Experimental Psychology, 38, 739-751.

Naveh-Benjamin, M., \& Jonides, J. (1984). Maintenance rehearsal: A two-component analysis. Journal of Experimental Psychology: Learning, Memory, and Cognition, 10, 369-385. https://doi.org/ 10.1037//0278-7393.10.3.369

Nelson, P. B., \& Soli, S. (2000). Acoustical barriers to learning: Children at risk in every classroom. Language, Speech and Hearing Services in Schools, 31, 356-361.

R Core Team. (2017). R: A language and environment for statistical computing. Vienna, Austria: R Foundation for Statistical Computing. Retrieved from www.R-project.org

Röer, J. P., Bell, R., Körner, U., \& Buchner, A. (2018). Equivalent auditory distraction in children and adults. Journal of Experimental 
Child Psychology, 172, 41-58. https://doi.org/10.1016/j.jecp.2018. 02.005

Röer, J. P., Körner, U., Buchner, A., \& Bell, R. (2017a). Attentional capture by taboo words: A functional view of auditory distraction. Emotion, 17, 740-750. https://doi.org/10.1037/emo0000274

Röer, J. P., Körner, U., Buchner, A., \& Bell, R. (2017b). Semantic priming by irrelevant speech. Psychonomic Bulletin \& Review, 24, 1205-1210.

Schafer, E.C. (2010). Speech perception in noise measures for children: A critical review and case studies. Journal of Educational Audiology, $16,4-15$.
Signorell, A. (2018). DescTools: Tools for descriptive statistics (R package version 0.99.24). Retrieved from https:/CRAN.R-project.org/ web/packages/DescTools/index.html

Turley-Ames, K. J., \& Whitfield, M. M. (2003). Strategy training and working memory task performance. Journal of Memory and Language, 49, 446-468. https://doi.org/10.1016/S0749-596X(03) 00095-0

Wickham, H., \& Henry, L. (2018). tidyr: Easily tidy data with "spread()" and "gather()" functions (R package version 0.8.1). Retrieved from https://CRAN.R-project.org/package=tidyr 